\title{
Uso do arco em "w" modificado no tratamento de mordida cruzada posterior simultâneo à manutenção de espaço anterior
}

\author{
Use of modified "w" arch in posterior crossbite treatment simultaneously with anterior \\ space maintenance
}

\begin{abstract}
Uso del arco "w" modificado en el tratamiento de la mordida cruzada posterior simultáneamente con el mantenimiento del espacio anterior
\end{abstract}

\begin{abstract}
Ana Carolina Leite de Arruda ${ }^{1}$, Fabiana Oliveira Henriques ${ }^{1}$, Anna Victória Costa Serique ${ }^{1}$, Pedro Aleixo Nogueira ${ }^{1}$, Suelly Maria Mendes Ribeiro ${ }^{1}$, Doris Kós Bulamarqui de Miranda ${ }^{1}$, Silvio Augusto Fernandes de Menezes ${ }^{1}$, Ricardo Roberto de Souza Fonseca ${ }^{2}$, Fabianny de Jesus Ribeiro Silva ${ }^{3}$, Jorge Sá Elias Nogueira ${ }^{1 *}$.
\end{abstract}

\section{RESUMO}

Objetivo: Apresentar um caso clínico de perda precoce (PP) de dente decíduo anterior associado a uma mordida cruzada posterior unilateral funcional direita (MCPUFD), cujo planejamento foi descruzamento da mordida com arco em "W" modificado concomitante à manutenção de espaço anterior. Detalhamento do caso: Paciente com quatro anos de idade, acompanhada da sua mãe, procurou atendimento de urgência em um pronto-socorro municipal, com trauma da face, apresentando ausência do elemento 51 após queda do beliche de altura com aproximadamente $1,5 \mathrm{~m}$, e ao ser examinada, constatou-se também MCPUFD. Para o tratamento, optou-se pelo arco em "W" modificado para o descruzamento da MCPUFD e manutenção estética do espaço anterior. Após noventa dias de tratamento, constatou-se linha média corrigida, boa relação dos caninos e das baterias posteriores e satisfação com aspecto estético pela paciente e responsáveis. Considerações finais: Conclui-se que a manutenção de espaço é de grande importância para a futura oclusão, além de contribuir para a melhora na autoestima e que o descruzamento da mordida será sempre um procedimento a ser executado o mais precoce possível.

Palavras-chave: Dente decíduo, Má oclusão, Mantenedor de espaço.

\begin{abstract}
Objective: To present a clinical case of early loss (PP) of anterior deciduos tooth associated with a right functional unilateral posterior crossbite (MCPUFD), whose planning was to uncross the bite with a modified "W" arch concomitant with space maintenance. Case Details: A four-year-old patient, accompained by his mother, seeks emergency care at a municipal emergency room, with trauma to the face, presents the lesions of element 51 after the fall of a height bunk of approximately $1,5 \mathrm{~m}$, and when being examined, MCPUFD was also found. For the treatment, the modified "W" arch was chosen to uncross the MCPUFD and aesthetic maintenance of the anterior space. After ninety days of treatment, a corrected midline was found, with a good ratio of canines and posterior batteries and satisfaction with aesthetic aspect by the patient and responsible. Final considerations: It is concluded that the maintenance of space is of great importance for future occlusion, ins addiction to contributing to the improvement in self-esteem and that uncrossing the bite will always be procedure to be performed as early as possible.
\end{abstract}

Keywords: Deciduous tooth, Malocclusion, Space maintainer.

\footnotetext{
${ }^{1}$ Centro Universitário do Estado do Pará (CESUPA), Belém-PA. *E-mail: jorge.nogueira@prof.cesupa.br

2 Universidade Federal do Pará (UFPA), Belém-PA.

${ }^{3}$ Escola Superior da Amazônia (ESAMAZ), Belém-PA.
}

SUBMETIDO EM: 5/2020

ACEITO EM: 6/2020

PUBLICADO EM: $8 / 2020$ 


\section{RESUMEN}

Objetivo: Presentar un caso clínico de pérdida temprana (PP) de diente deciduo anterior asociado con una mordida cruzada posterior funcional unilateral directa (MCPUFD), cuyo plano fue removido con una mordida en el arco "W", modificado simultáneamente para mantener el espacio. Detalles del caso: un paciente de cuatro años acompañado de su madre, buscó atención de emergencia en una sala de emergencias municipal, con traumatismo facial, con la ausencia del elemento 51 después de caer de la litera a una altura de aproximadamente 1,5m, cuando fue examinado, MCPUFD tambén se encontró. Para el tratamiento, se eligió el arco "w" modificado para descruzae el MCPUFD y el mantenimiento estético del espacio anterior. Después de noventa días de tratamiento, se encontró una línea media corregida, una buena proporción de los caninos y las baterías posteriores y la satisfacción con los aspectos estéticos por parte del paciente y los tutores. Consideraciones finales: Concluye que mantener el espacio es de gran importancia para la oclusion futura, además de contribuir a la pérdida de la autoestima y que revelar la mordida siempre será un procedimiento que se realizará o posiblemente antes.

Palabras clave: Diente deciduo, Maloclusión, Mantenedor de espacio.

\section{INTRODUÇÃO}

Entende-se por PP quando ocorre a saída de um elemento dentário do seu alvéolo antes do tempo de sua esfoliação normal (GONÇALVES LM, et al., 2013). A perda prematura de dentes decíduos ocorre geralmente por restaurações inadequadas, reabsorção prematura das raízes dentárias, odontodisplasia, e principalmente por trauma e lesões de cárie (OLIVEIRA LRR, et al., 2017). A ausência precoce interfere no crescimento craniofacial e pode causar problemas na oclusão, como a diminuição do perímetro do arco, migração dos dentes vizinhos, desenvolvimento de hábitos deletérios e, como resultado, alterações na função fonética, respiratória, mastigatória e estética. Logo, torna-se óbvia a importância de preservá-lo até a sua esfoliação natural (GONÇALVES LM, et al., 2013; GUIMARÃES CA e OLIVEIRA RCG, 2017; NOBREGA ML, et al., 2018).

As mal oclusões podem ser caracterizadas como a disposição que os dentes assumem nos arcos dentários e a relação destes com as bases ósseas de forma desarmônica (ARAUJO CRUZ, JH, et al., 2019). Estudos têm relacionado a mordida cruzada posterior $(\mathrm{MCP})$ à presença de hábitos orais deletérios, a citar: distúrbios miofuncionais, orofaciais e respiratórios (GRABOWSKI R, et al., 2007). Sendo importante o correto diagnóstico da MCP se dentoalveolar, esquelética ou funcional (MOYERS RE, 1991). Nesse sentido, a odontopediatria exerce papel importante na prevenção e correção de mal oclusões visando otimizar o crescimento e o desenvolvimento dentofacial (MOSTAFIZ W, 2019).

A reabilitação estética-funcional é importante para casos onde há tanto a perda quanto problemas de maloclusão, visto que o tratamento precoce impede a interposição lingual, a movimentação dos dentes e maloclusões na dentição permanente. Portanto, a seleção do aparelho deve tomar como base a idade do paciente, grau de cooperação, anseios da criança e de seus responsáveis (GUIMARÃES CA e OLIVEIRA RCG, 2017; ARAUJO CRUZ JH, et al., 2019; DAINEZI VB, et al., 2015; OTA CM, et al., 2014).

O objetivo do trabalho é apresentar o tratamento proposto para um caso clínico de PP de dente decíduo anterior associado a uma MCPUFD onde o planejamento foi a utilização de um aparelho em "w" modificado, como alternativa de tratamento para o descruzamento da mordida cruzada posterior simultâneo a manutenção de espaço anterior.

\section{DETALHAMENTO DE CASO}

Paciente 4 anos de idade, gênero feminino, compareceu ao Hospital de Pronto Socorro Municipal de Belém Mario Pinotti, na madrugada, acompanhada pela mãe após queda do beliche (aproximadamente 1,5m de altura), envolvendo cavidade bucal. Cumprido o protocolo de avaliação médica, para exclusão de trauma cranioencefálico (TCE), descartando lesões de maior gravidade, devido apresentação clínica de paciente consciente e orientada, bom estado geral, pupilas isocóricas e foto reagentes, escore na escala de coma de 
Glasgow 15 (Jain e Iverson, 2020). Após esta avaliação, foi encaminhada ao cirurgião dentista de plantão que constatou após exame intra e extrabucal ausência clínica do elemento 51 , lesão de tecido mole do referido dente (Figura 1A). Alvéolo vazio pela avulsão do elemento 51, constatado pelo exame radiográfico (Figura 1B). Durante anamnese, a mãe afirmou que o dente havia saído e se encontrava guardado em um recipiente plástico na residência. Recebeu alta para tratamento ambulatorial acompanhada de receita analgésica, orientação de higiene e alimentação.

A menor não contribuiu para realização de documentação ortodôntica e o diagnóstico fundamentou-se pelo exame clínico onde mostrou simetria do arco superior e inclinação do canino superior direito, o qual provocava desvio mandibular para acomodação da intercuspidação, comprovando o desvio da linha média (Figura 2A). Posição desfavorável com mesialização do canino superior direito e elementos superiores posteriores cruzados (Figura 2B). Boa relação do canino e elementos superiores esquerdos (Figura 2C). Nesse momento, optou-se pelo descruzamento da MCPUFD utilizando-se o arco em "W" modificado com fio ortodôntico $0,9 \mathrm{~mm}$ para descruzamento da mordida e adaptação de elemento dentário estético para o decíduo.

Figura 1 - 1A: Imagem inicial após trauma; 1B: Imagem radiográfica confirmando avulsão do 51, monstrando alvéolo vazio.

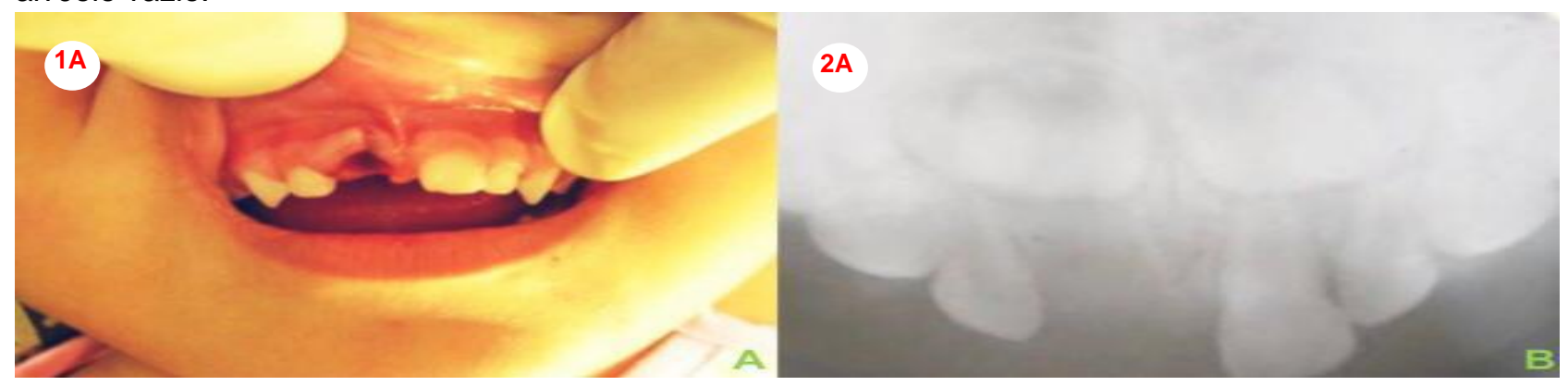

Fonte: Nogueira JSE, et al., 2020.

Figura 2 - 2A: Evidenciando desvio de linha média; 2B: Mesialização do elemento 53/ 2C: Boa relação de canino superior e bateria superior esquerda; 2D: Arco em "W" modificado cimentado.

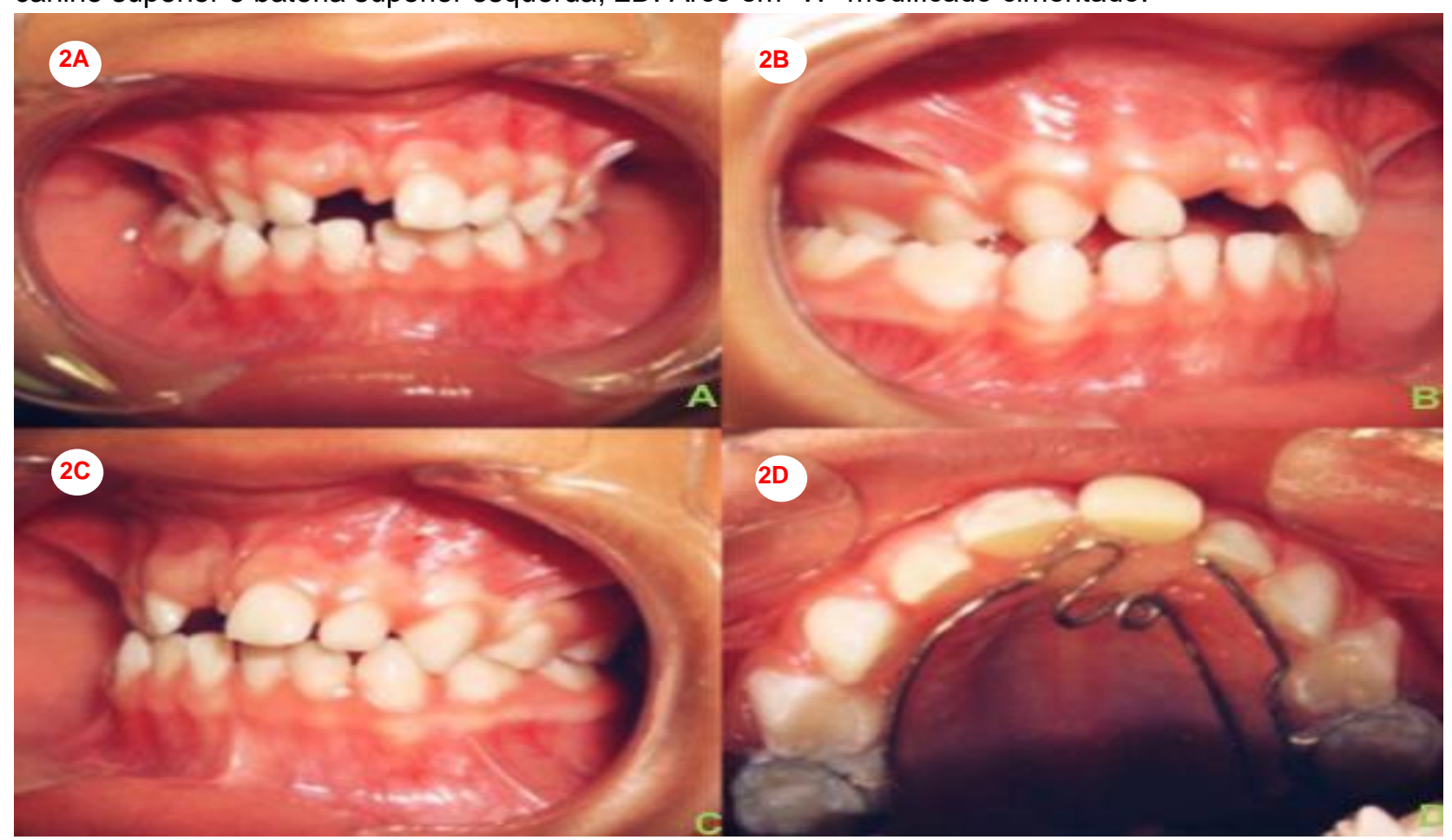

Fonte: Nogueira JSE, et al., 2020. 
Após 30 dias da cimentação, em consulta de primeiro controle, mostrou boa relação de linha média e intercuspidação favorável da bateria posterior direita (Figura 3A). Controle radiográfico oclusal (Figura 3B). E boa relação dos caninos direito e esquerdo (Figura 3C e 3D respectivamente). Sessenta dias após cimentação, paciente mostra linha média corrigida (Figura 4A). Controle radiográfico oclusal (Figura 4B). Boa relação dos caninos e das baterias posteriores (Figura 4C e 4D).

Figura 3 - 3A: Primeiro controle 30 dias após, evidenciando melhora na linha média; 3B: Controle radiográfico; 3C: Vista lateral direita; 3D: Vista lateral esquerda.

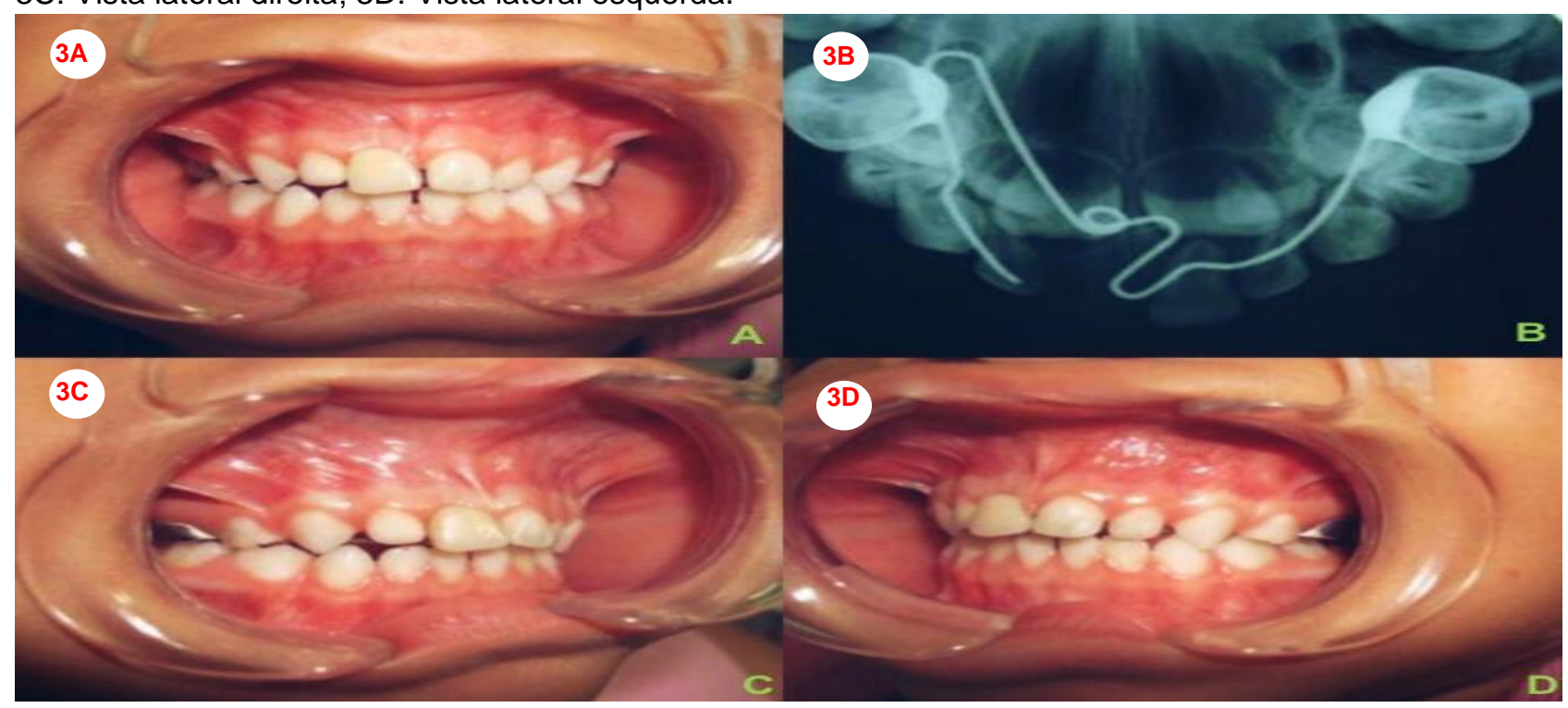

Fonte: Nogueira JSE, et al., 2020.

Noventa dias após cimentação, optou-se pela remoção do arco em "W" para confecção de arco palatino de Nance modificado para contenção (Figura 5A). Fixação do elemento 51 como mantenedor estético (Figura 5B). Vista frontal inicial (Figura 5C). Final do caso concluído para controle (Figura 5D).

Figura 4 - 4A: Controle após 60 dias evidenciando boa relação oclusal anterior com linha média e linha média coincidente; 4B: Controle radiográfico; 4C: Visão direita; 4D: Visão esquerda.

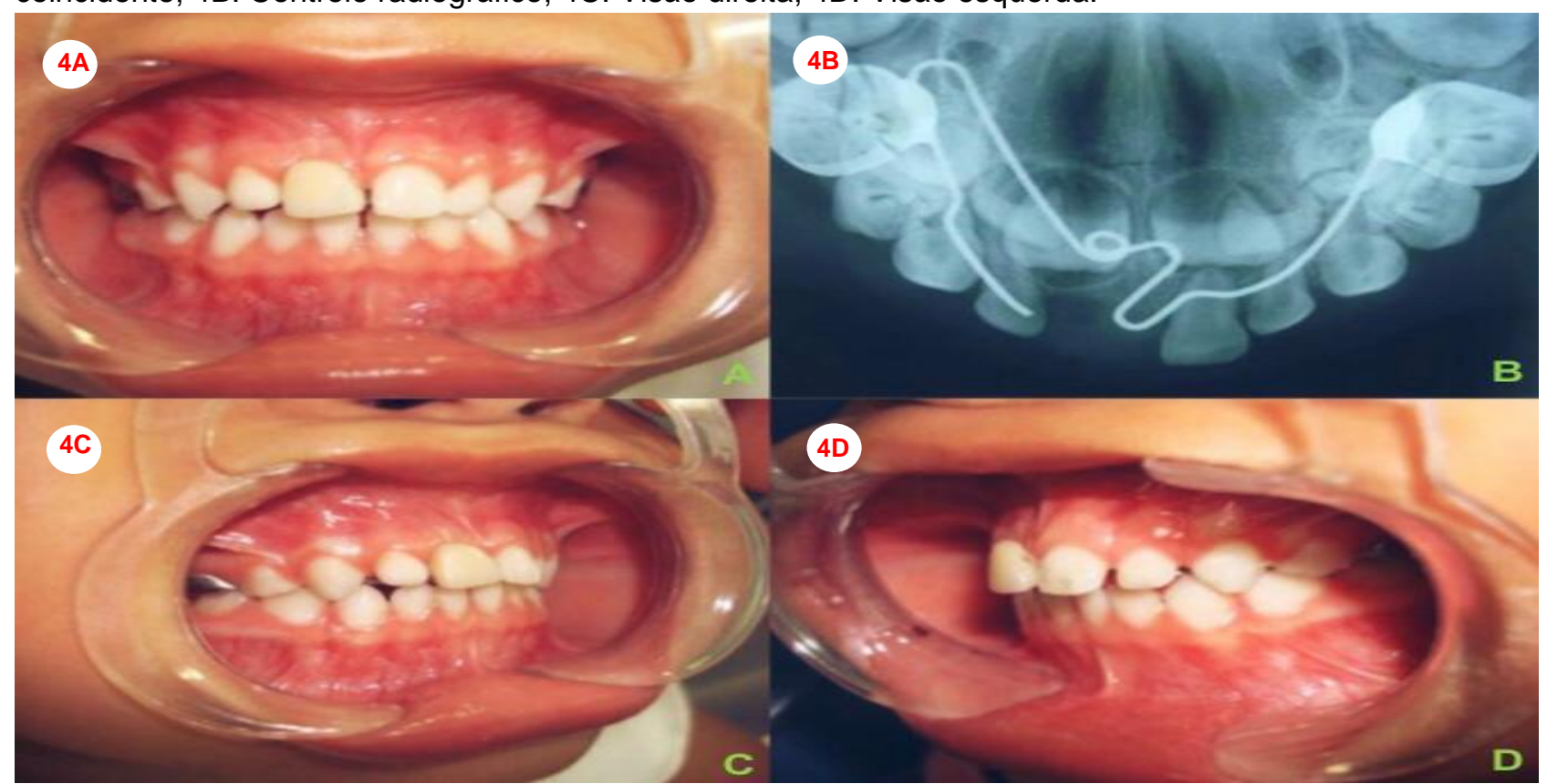

Fonte: Nogueira JSE, et al., 2020. 
Figura 5 - 5A: Confecção do arco palatino de Nance modificado; 5B: Visão palatina; 5C: Visão frontal pré expansão; 5D: Visão final.

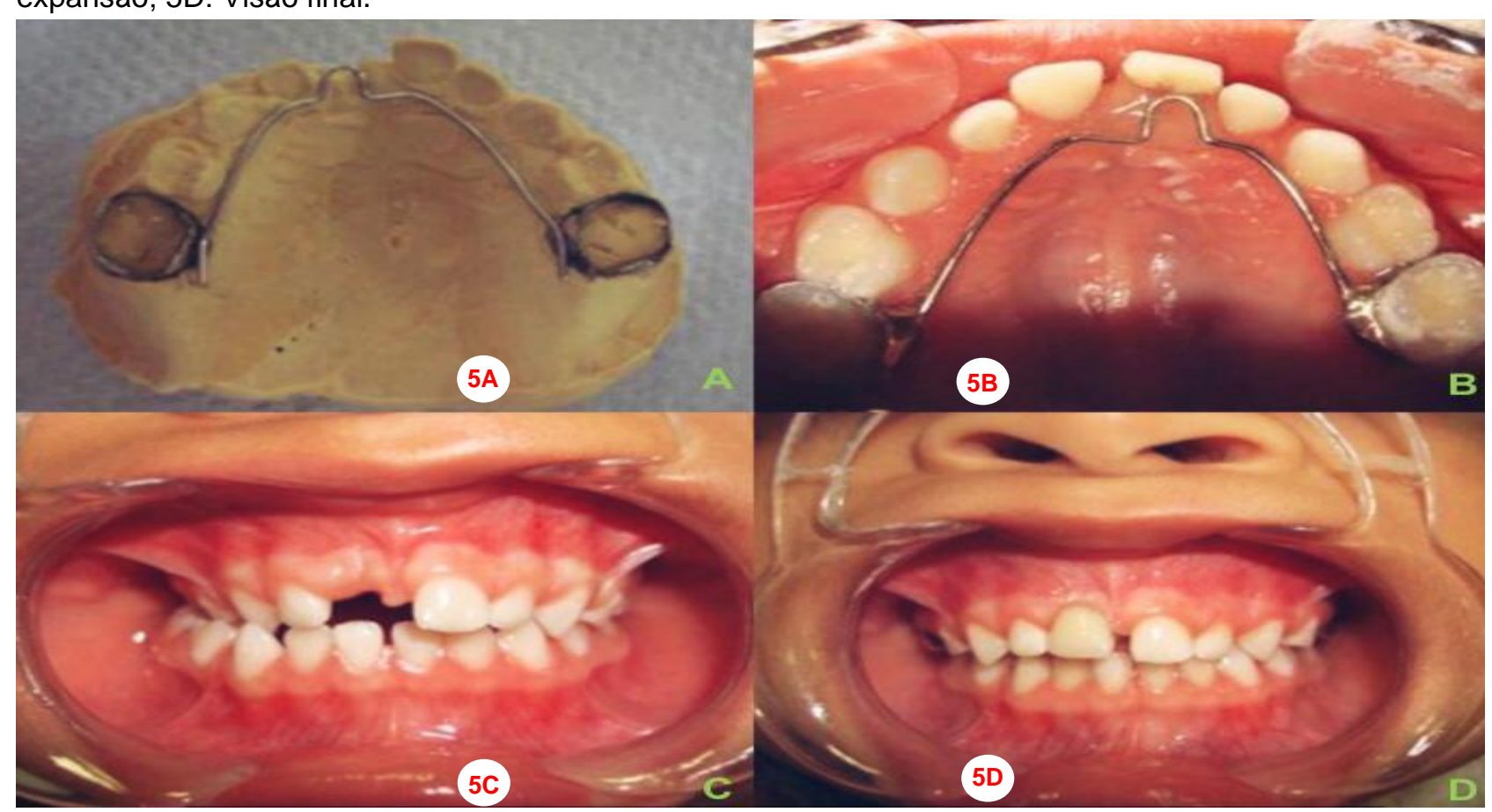

Fonte: Nogueira JSE, et al., 2020.

\section{DISCUSSÃO}

O atendimento de urgência em avulsão é sempre fundamentado em reimplantar o quanto antes o elemento dentário permanente para aumentar a chance de mantê-lo na cavidade bucal, o que torna sombrio o prognóstico com dentes decíduos pela possibilidade de causar infecção, anquilose e danos ao sucessor (CANEVER FF, et al., 2019). Apesar de que para alguns o reimplante seja viável (POLUHA RL, et al., 2016; MACLEOD SP e RUDD TC, 2012). Nossa conduta foi não reimplantar e o passo seguinte fundamentou-se no tratamento sintomático, condicionamento psicológico, reabilitação e acompanhamento das repercussões para a dentição decídua e permanente.

O exame clínico pediátrico frequentemente é ainda mais difícil quando se torna necessário após a ocorrência de trauma, principalmente quando há envolvimento de sangue (OLIVEIRA E, et al., 2009). As crianças dificilmente são capazes de fornecer detalhes sobre o mecanismo do trauma e geralmente são pouco colaborativas e muito amedrontadas, exigindo experiência e paciência por parte do profissional que necessita realizar sua observação, palpação e exame funcional da área atingida pelo trauma. Assim como em adultos, radiografias de face fazem parte do protocolo de atendimento e avaliação de diversas especialidades médicas a citar: pediatria, traumatologia e neurologia, sendo posteriormente encaminhado à odontologia. Este protocolo contribui para um tempo grande de permanência hospitalar levando a criança a contribuir negativamente com a assistência (BARROS JJ e SOUZA LCM, 2000).

A PP de decíduos anteriores é um problema frequente na odontopediatria que pode provocar perda da eficiência mastigatória, distúrbios fonéticos, desequilíbrio oclusal, comprometimento estético, e como resultado de tudo, os problemas psicoemocionais (OLIVEIRA LRR, et al., 2017; GUIMARÃES CA e OLIVEIRA RCG, 2017; NOBREGA ML, et al., 2018). Korytnicki D, et al. (1994); afirmam não existir regras para a instalação de mantenedor de espaço (ME) para a região anterossuperior, devendo-se analisar atenciosamente cada caso, levando em consideração os seguintes fatores: tipo de dente perdido, idade da criança, estágio de desenvolvimento que a criança se encontra, características do arco dentário e presença de hábitos deletérios (KORYTNICKI D, et al., 1994; GUIMARÃES CA e OLIVEIRA RCG, 2017). 
Um aspecto importante a considerar está na decisão em manter ou não o espaço frente à PP de dente decíduo anterior. Korytnicky D, et al. (1994); afirmam que a PP dos dentes decíduos na região anterossuperior, após o estabelecimento da oclusão, não necessita de aparelho $\mathrm{ME}$, pois não há relatos de fechamento de espaço se os caninos já estiverem irrompidos. Lopez BMM, et al. (2003); reforçam que a PP de dente decíduo anterior pode ocasionar o deslocamento dos dentes adjacentes e a extrusão dos dentes antagonistas dependendo do estágio de formação radicular do sucessor. Guedes Pinto AC (1991); acrescenta também a possibilidade de giroverssão. Kisling E e Hoffding J (1979); reforçam ainda que a decisão dependerá também do espaço que a arcada dentária contém, do padrão de erupção, intercuspidação, das características esqueléticas, função muscular e condições periodontais.

Wanderley MT, et al. (1998) e Galindo VAC, et al. (2003) consideram indispensáveis o uso dos ME para preservar o espaço deixado pelo dente perdido prematuramente, permitindo que os dentes permanentes sucessores erupcionem na posição correta, fazendo a manutenção do perímetro do arco, pois a integridade dos dentes e das arcadas é fundamental para a manutenção da fonética, oclusão, estética e do bem-estar emocional da criança.

Pelo exposto, baseando-se no fato de nossa paciente apresentar arco tipo II de Baume LJ (1950); associado à presença de MCP incluindo o canino (53), nossa decisão foi em manter o espaço reforçada pela necessidade emocional. Frente à impossibilidade de obtenção de documentação ortodôntica completa devido à falta de colaboração e pela inequívoca indicação da colocação do ME, que era uma exigência da paciente, aproveitamos o momento para o concomitante descruzamento da MCP após avaliação clínica, análise de radiografias ocluais e dos modelos em gesso.

A literatura evidencia que o tratamento da MCP deve ser iniciado o mais cedo possível, visto que a autocorreção durante a transição da dentição decídua para a permanente é pouco provável ou até impossível (GARIB DG e SANTOS PB, 2013). Sendo assim, o tratamento interceptivo consiste na correção das discrepâncias esqueléticas, musculares ou dentoalveolares, pois irá redirecionar a posição dos germes dos dentes permanentes, permitir uma boa relação entre as bases apicais, corrigir a função mastigatória, eliminar posições inadequadas da articulação temporomandibular tornando simétrica a relação da cabeça da mandíbula nas fossas articulares (BATISTA ER e SANTOS DCL, 2017).

Quanto a realização do tratamento correto e escolha do tipo de aparelho a ser utilizado, é importante a realização de um bom diagnóstico, levando em consideração os tipos de MCP. O tratamento da MCP pode ser realizado com aparelho removível ou fixos, a citar: placa de Hawley (removível) com parafusos expansores destinados às MCP dentárias e os fixos com a utilização de quadrihélice ou arco em "W" às funcionais (LOPES CDF e SILVA RND, 2015). Hass e Hyrax, as esqueléticas (BATISTA ER e SANTOS DCL, 2017; FERNANDES BDA, 2018). O arco em "W" possui vantagens que fazem dele um dos aparelhos mais utilizados para 0 tratamento da mordida cruzada posterior funcional (MCPF), pois é flexível ao ajuste, fácil de ser higienizado e por ser um aparelho fixo, pode ser ancorado nas bandas dos molares permanentes ou decíduos e ainda independe de colaboração do paciente infantil. (FERNANDES BDA, 2018; PEREIRA RA, et al., 2017).

Em relação à MCP, por acreditar que possa ser transferida para dentição permanente, autores incentivam o tratamento o quanto antes, com o intuito de estabelecer um ambiente favorável para o correto crescimento dos ossos da face (ARAUJO CRUZ JH, et al., 2019; BATISTA ER e SANTOS DCL, 2017). Evidências cientificas reforçam que, se uma MCPF deixada sem tratamento, poderá ter efeitos deletérios no desenvolvimento e na função das articulações temporomandibulares (Uysal T, et al., 2009). A assimetria facial e o desvio da linha média mandibular em relação ao lado da mordida cruzada podem persistir, e a adaptação subsequente da neuromusculatura à posição mandibular adquirida pode causar crescimento mandibular assimétrico, desarmonia facial e mordida cruzada esquelética grave na dentição permanente. Vários estudos documentaram que uma posição do côndilo mais simétrica foi alcançada após o tratamento da MCP. Como a correção espontânea da MCP é rara, o tratamento precoce tem sido recomendado (THILANDER B, et al., 1984 e LINDNER UMA, 1989). 
A revisão sistemática também reforça que MCP afeta o desenvolvimento harmonioso das estruturas orofaciais (KARIN SM, et al., 2018). No que se refere à eleição dos aparelhos ortodônticos para tratamento das MCPF não há unanimidade entre os autores: Ribeiro Júnior HC, et al. (2004) recomendam desgaste seletivo para eliminar interferências, conseguindo então levar o paciente à uma correta oclusão. Figueiredo MA, et al. (2007) demonstraram que também é eficaz o tratamento com o aparelho quadrihélice. Garbin AJI, et al. (2016) e Chibinski ACR, et al. (2005) recomendam a intervenção com a utilização de Pistas diretas de Planas, nivelando a linha média, estabelecendo padrão oclusal harmônico, proporcionando o desenvolvimento da face e da dentição dentro dos padrões de normalidade. Nossa opção pelo arco em "W" fundamentou-se pelo fato da paciente apresentar envolvimento de vários elementos dentários, onde apenas o desgaste do canino não estaria indicado.

Tendo em vista a análise da literatura, discussão e o resultado clínico, concluímos que a manutenção de espaço estética não deve ser postergada frente ao desejo da paciente; a MCP deve ser trata o quanto antes, a fim de proporcionar um bom crescimento e desenvolvimento crânio facial; não há qualquer empecilho no tratamento concomitante de uma MCP e na manutenção de espaço. Dessa forma, o planejamento do caso incluiu o uso de dois aparelhos ortodônticos fixos, de baixo custo, fácil higienização e que não necessitaram de grande contribuição da paciente. Mediante a isso, o dentista deve estar devidamente preparado para que possa identificar a MCP e a PP, mas também para chegar a um diagnóstico preciso, correto e no momento certo.

\section{REFERÊNCIAS}

1. ARAUJO CRUZ JH, et al. Mordida cruzada posterior: um enfoque à epidemiologia, etiologia, diagnóstico e tratamento. Archives of health investigation, 2019; 8(3).

2. BARROS JJ, SOUZA LCM. Traumatismo buco-maxilo-facial. 2a. ed. São Paulo: Roca; 2000.

3. BATISTA ER, SANTOS DCL. Mordida cruzada posterior em dentição mista. Rev. Odontol. Univ. Cid. São Paulo, 2017; 29(1), 66-74.

4. BAUME LJ. Physiological tooth migration and its significance for the development of occlusion I: the biogenetic course of the deciduous dentition. J Dent Res, 1950; 29(2): 123-132.

5. CANEVER FF, et al. Reimplante de dente decíduo: revisão bibliográfica integrativa. Rev. Odontol. Univ. Cid. São Paulo, 2019; $31(2): 168-76$.

6. CHIBINSKI ACR, et al. "Pistas diretas planas: terapia ortopédica para correção de mordida cruzada funcional." R Clin Ortodon Dental Press, 2005; 64-72.

7. DAINEZI VB, et. al. Reabilitação estética e funcional na primeira infância: relato de caso. Rev Assoc Paul Cir Dent, 2015; 69(4), 387-393.

8. FERNANDES BDA, Etiologia e tratamento da mordida cruzada posterior na dentição decídua e mista. Dissertação (Mestrado em Medicina Dentária) - Instituto Universitário Egas Moniz, 2018.

9. FIGUEIREDO MA, et al. Tratamento precoce da mordida cruzada posterior com o Quadrihélice de encaixe. Rev clín ortodon Dental Press, 2007; 5(6):83-94.

10. GALINDO VAC, et al. Aparelho mantenedor de espaço estético e funcional na dentição decídua. J Bras Odontopediatria Odontol Bebê, 2003; 6(30):99-104.

11. GARBIN AJI, et al. A utilização da Pista Direta de Planas no tratamento precoce da mordida cruzada posterior: relato de caso. Arch. Heal. Inv, 2016; 5(4).

12. GARIB DG, SANTOS PB, Mordida Cruzada Posterior e Anterior. Cap. 6. In: Janson, G. e col. Introdução à Ortodontia. 1a Ed. São Paulo: Artes Médicas, p. 82-91, 2013. 159 p.

13. GONÇALVES LM, et al. Uso de prótese fixa adesiva como mantenedor de espaço em dentes anteriores decíduos: um relato de caso. Arch. Ora.I Res, 2013; 9(1).

14. GRABOWSKI R, et al. Relationship between occlusal findings and orofacial myofunctional status in primary and mixed dentition. Part I: Prevalence of malocclusions. J Orofac Orthop. 2007;68(1):26- 37.

15. GUEDES PINTO AC. Odontopediatria. $3^{\text {a }}$ ed. São Paulo: Santos, 1991.

16. GUIMARÃES CA, OLIVEIRA RCG. Perda precoce de dentes decíduos: relato de caso clínico. Rev. UNINGÁ Review. 2017; 29(n.2): 28-33.

17. JAIN S, IVERSON LM. Escala de coma de Glasgow. In: StatPearls [Internet]. Ilha do Tesouro (FL): StatPearls Publishing; 2020 Jan-. Disponível em: https://www.ncbi.nlm.nih.gov/books/NBK513298/

18. KARIN SM, et al. O efeito da sucção de chupeta nas estruturas orofaciais: uma revisão sistemática da literatura. Prog. Ortho, 2018; 19(8).

19. KISLING E, HOFFDING J. Premature loss of primary teeth: art III, drifting patterns for different types of teeth loss of adjoining teeth. J Dent Child, 1979;46:34-38. 
20. KORYTNICKI D, et al. Consequências e tratamento das perdas precoces de dentes decíduos. Rev Assoc Paul Cir Dent, 1994; mai-jun; 48(3):1323-9.

21. LINDNER UMA. Estudo longitudinal sobre o efeito do tratamento interceptivo precoce em crianças de 4 anos com mordida cruzada unilateral. Scand J Dent Res. 1989;97(5): 432-438.

22. LOPEZ BMM, et al. Importancia de los dientes temporales. Su cronología de erupción. Rev. Pedia. Aten Prima, 2003;5(19): 439-445

23. LOPES CDF, SILVA RND. Tratamento interceptativo da mordida cruzada posterior. Trabalho de Conclusão de Curso de Odontologia - Universidade Tiradentes, Aracaju, 2015.

24. MACLEOD SP, RUDD TC. Update on the management of dentoalveolar trauma. Curr. opin. otol. head. neck. sur, 2012; 20(4):318-24.

25. MOSTAFIZ W. Compend Contin Educ Dente; 2019; 40(3): 149-154; quiz 155.

26. MOYERS RE. Classificação e terminologia da má-oclusão. Ortodontia. 4. Edição. Rio de Janeiro: Guanabara Koogan, 1991.

27. NOBREGA ML, et al. Implicações da perda precoce em odontopediatria. Rev. Pró-UniverSUS, Vassouras, 2018; 9(1): 61-67.

28. OLIVEIRA E, et al. Fraturas de face na infância: experiência em 369 casos. Rev Bras Cir Craniomaxilofac, 2009; 12(4):133-137.

29. OLIVEIRA LRR, et al. Uso de mantenedor de espaço em região anterior: revisão de literatura e relato de caso clínico. Arch Neurol, 2017.

30. OTA CM, et al. Mantenedor fixo estético-funcional como tratamento para perda precoce de dentes decíduos anteriores. Rev Assoc Paul Cir Dent, 2014;68(4): 307-311

31. PEREIRA RA, et. al. Mordida Cruzada Posterior. Rev. Red. Ciud. Saüde. $2017 ; 11(2)$.

32. POLUHA RL, et al. Reimplante de dentes decíduos: indicações e contra-indicações. Arch Health Invest. 2016; $5(3): 140-3$

33. RIBEIRO JÚNIOR HC, et al. Tratamento precoce da mordida cruzada posterior functional. Arq Odontol, $2004 ; 40$ (2): 149-158.

34. THILANDER B, et al. O efeito do tratamento interceptivo precoce em crianças com mordida cruzadaposterior. Eur $\mathrm{J}$ Orthod. 1984;6(11):25-34.

35. UYSAL T, et al. Assimetria vertical condilar e ramal em pacientes com mordida cruzada posterior unilateral e bilateral e uma amostra de oclusão normal. Am J Orthod Dentofacial Orthop, 2009; 136(11):37.-43.

36. WANDERLEY MT, et al. Recursos protéticos em odontopediatria. In: Corrêa MSNP. Odontopediatria na primeira infância. São Paulo: Santos; 1998; 497-512. 